\title{
Laboreal
}

Volume $11 \mathrm{~N}^{\circ} 1$ | 2015

Modos de vida e trabalho

\section{Un modelo de investigación colectiva e internacional sobre la seguridad: contribución a la historia de las investigaciones sobre seguridad en el trabajo: resumen de la investigación CECA sobre la seguridad en la siderurgia (1962-1966)}

Um modelo de investigação coletiva e internacional acerca da segurança:

Contribuição para a história das pesquisas sobre segurança do trabalho: sinopse da pesquisa da CECA sobre a segurança na siderurgia (1962-1966)

Un modèle de recherche collective et internationale sur la sécurité: Contribution à l'histoire des recherches sur la sécurité du travail : un aperçu de la Recherche de la CECA sur la sécurité dans la sidérurgie (1962-1966)

A group and international research model about safety: Contribution for the history of researches on safety at work: an overview of the ESCS research on safety in the steel industry (1962-1966)

Jacques Leplat

\section{OpenEdition}

Journals

Edición electrónica

URL: http://journals.openedition.org/laboreal/4433

DOI: $10.4000 /$ laboreal.4433

ISSN: 1646-5237

Editor

Universidade do Porto

Referencia electrónica

Jacques Leplat, « Un modelo de investigación colectiva e internacional sobre la seguridad: contribución a la historia de las investigaciones sobre seguridad en el trabajo: resumen de la investigación CECA sobre la seguridad en la siderurgia (1962-1966) », Laboreal [En línea], Volume 11 N01 | 2015, Publicado el 01 julio 2015, consultado el 24 septiembre 2020. URL : http:// journals.openedition.org/laboreal/4433; DOI : https://doi.org/10.4000/laboreal.4433

Este documento fue generado automáticamente el 24 septiembre 2020

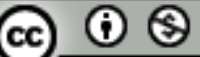

Laboreal está licenciado com uma Licença Creative Commons - Atribuição-NãoComercial 4.0 Internacional. 


\section{Un modelo de investigación} colectiva e internacional sobre la seguridad: contribución a la historia de las investigaciones sobre seguridad en el trabajo: resumen de la investigación CECA sobre la seguridad en la siderurgia (1962-1966)

Um modelo de investigação coletiva e internacional acerca da segurança: Contribuição para a história das pesquisas sobre segurança do trabalho: sinopse da pesquisa da CECA sobre a segurança na siderurgia (1962-1966)

Un modèle de recherche collective et internationale sur la sécurité: Contribution à l'histoire des recherches sur la sécurité du travail : un aperçu de la Recherche de la CECA sur la sécurité dans la sidérurgie (1962-1966)

A group and international research model about safety: Contribution for the history of researches on safety at work: an overview of the ESCS research on safety in the steel industry (1962-1966)

Jacques Leplat 


\title{
REFERENCIA
}

CECA. (1969). Recherche communautaire sur la sécurité des mines et la sidérurgie (1962-1966). Communauté Européenne du charbon et de l'Acier (CECA). Etudes de physiologie et de psychologie du travail, $n^{\circ} 4,1969$, pp. 13-24.

\section{NOTA DEL EDITOR}

\author{
http://dx.doi.org/10.15667/laborealxi0115jles
}

1 Las investigaciones sobre la seguridad de las personas en los diversos trabajos a los cuales se las ha asociado poseen una larga historia a menudo mencionada en las numerosas obras generales consagradas a la seguridad. La psicología moderna también refleja esta preocupación, como lo atestigua sobre todo la bibliografía de la llamada psicología aplicada desde finales del siglo XIX. Francia ha realizado importantes contribuciones a estas investigaciones, tal como lo atestiguan sobre todo los trabajos llevados a cabo o dirigidos por Lahy, Pacaud, Reuchlin y Faverge en el siglo XX. Las investigaciones iniciales se orientaron sobre todo hacia la psicología diferencial, haciendo un uso muy extendido de los tests psicológicos. La mejora de la seguridad se investigó desde la perspectiva de la mejora de la adaptación de las personas a sus tareas. Sin embargo, tras la segunda guerra mundial, se desarrollaron corrientes de investigación encaminadas a ampliar el campo de la psicología del trabajo hacia el estudio de la seguridad: en pocas palabras, existía un interés no ya solo por la adaptación de la persona a su trabajo, sino también por la adaptación del trabajo a la persona y, finalmente, por el diseño de situaciones laborales que generaran una mejor articulación de las condiciones humanas, organizativas, técnicas y sociales adecuadas para crear un entorno laboral más seguro. El texto aquí presentado se inscribe en este periodo de transición y permite comprender mejor la naturaleza y las modalidades de las evoluciones posteriores.

2 La breve presentación de este artículo se organiza en dos partes. La primera precisa el marco en el que se inscribe y la segunda ofrece algunas justificaciones a la elección de la parte del documento que se ha elegido para su publicación en este número de Laboreal.

\section{El contexto del texto}

El texto aquí presentado hace referencia a las investigaciones llevadas a cabo en el marco de un extenso programa titulado "Recherche communautaire sur la sécurité dans les mines et la sidérurgie" (Investigación comunitaria sobre la seguridad en las minas y la siderurgia): se refiere a la parte relativa a la siderurgia, siendo distinta la que concierne a las minas, además de que la llevó a cabo otro equipo. Las investigaciones incluidas en estos documentos se refieren a "acciones llevadas a cabo por la Alta Autoridad de la CECA (Comunidad Europea del Carbón y del Acero) para el progreso de las investigaciones sobre la prevención de los accidentes" (pág. 5)ํㅗ ${ }^{1}$ Su concepción es la 
continuación de anteriores investigaciones procedentes de un programa elaborado en 1959. Al igual que aquel programa, este otro fue preparado a través de intercambios entre organizaciones profesionales e institutos de investigación de los cinco países miembros de la CECA: Alemania, Francia, Italia, Bélgica y Países Bajos. Faverge desempeñó un papel importante en la elaboración de este programa que fue finalmente precisado colectivamente y cuya realización tuvo lugar entre 1962 y 1966. Se trataba de una investigación verdaderamente colectiva jalonada periódicamente de reuniones de todos los equipos en Luxemburgo durante dos días en los que se debatía, garantizando así una coordinación del desarrollo de las investigaciones. También se organizaron visitas a los lugares donde se estaban desarrollando las investigaciones. Al término de las investigaciones, se estableció un informe de síntesis para cada equipo nacional y se designó a un coordinador para la redacción del informe de síntesis general. Es de dicho informe de 231 páginas del que se ha extraído el texto del presente artículo.

Los campos de investigación elegidos por cada equipo eran diferentes.

- Alemania: laminadoras, fundiciones, líneas de acabado.

- Bélgica: altos hornos.

- Francia: transportes.

- Italia: laminadoras.

- Países Bajos: reparaciones.

5 Los títulos de los diferentes capítulos del informe muestran con claridad la diversidad de temas abordados.

- Recopilación y explotación preliminar de las informaciones sobre la seguridad y el trabajo.

- Organización del trabajo.

- La seguridad y el grupo de trabajo.

- Instrumentos y condiciones laborales.

- Características individuales.

- Propuestas concretas para mejorar la prevención de accidentes.

\section{Justificación y contenido del texto.}

El documento del que se ha extraído el presente artículo informa de los trabajos realizados en el transcurso de la década de 1960, en una época en la que el estudio de los accidentes todavía se centraba en las características individuales de las personas implicadas en los accidentes. Desde finales del siglo XIX, esta concepción estuvo vigente y sostenida por múltiples publicaciones, tal como lo atestigua, por ejemplo, una bibliografía de los trabajos de Lahy (1872-1943) establecida por Turbiaux (1982-3). El "factor humano" era explotado sobre todo para la selección de diferentes tipos de operarios, en concreto maquinistas de tren y operadores de otras máquinas. Un texto particularmente bien documentado publicado por Lahy, y Korngold Pacaud en 1936, titulado "Recherches expérimentales sur les causes psychologiques des accidents du travail" (Investigaciones experimentales sobre las causas psicológicas de los accidentes laborales) ilustra bien esta concepción del accidente. Veamos, por ejemplo, algunos elementos de los títulos de sus diversas partes: el factor humano en general y el factor individual en los accidentes laborales, la tesis de la "predisposición", estudio de la fatiga, y la práctica de la selección preventiva. En esta misma publicación, podemos leer que, en 1928, en el congreso internacional de psicotecnia, la plaga de los accidentes se comparó con la de las enfermedades y se dijo que requería una profilaxis análoga. 
7 Pero la investigación CECA sería concebida con una perspectiva totalmente diferente. Su documento preparatorio, que había sido preparado por un equipo que englobaba a representantes de sectores profesional, científico y gubernamental, "expresaba el deseo de que en un estudio que abarcaba un campo tan vasto se prefiriera un estudio centrado en los efectivos limitados, elegidos en una unidad operativa determinada" (pág. 11). Al mismo tiempo se destacaba "la necesidad de un análisis muy concreto sobre las condiciones de la actividad y de un esfuerzo por vincular el accidente a las propias situaciones laborales" (id.). También se requería que dicha limitación del ámbito de ampliación "oriente a esta hacia un verdadero análisis de los mecanismos generadores de accidentes y que permita así extraer aplicaciones precisas del plan de la seguridad laboral" (id.). Las investigaciones referidas en el presente artículo observan estos principios en los que, en la época, radicaba su originalidad y que expresaban una ruptura con las investigaciones anteriores. Tras las investigaciones centradas en el papel del operario, siguieron investigaciones centradas en las situaciones laborales y la integración de sus distintas dimensiones: psicológica, técnica, organizativa, sociológica y social. En otras publicaciones de la CECA de la época, encontramos las mismas características. Por ejemplo, una publicación colectiva de la misma colección se titula "Fiabilité et sécurité" (Fiabilidad y seguridad) (1972) e incluye textos sintéticos que hacen hincapié tanto en los incidentes como en los accidentes, además de otorgar una importancia creciente a las condiciones organizativas. Todos estos trabajos contribuirían a la renovación de los marcos de investigación sobre la seguridad laboral.

Como el espacio reservado para el presente artículo era limitado, había que elegir un extracto de la obra sobre la investigación comunitaria que poseyera los rasgos originales más característicos. Nos pareció que la parte introductoria del informe, titulada "Considérations générales sur les travaux de la recherche communautaire" (Consideraciones generales sobre los trabajos de la investigación comunitaria), aportaba los elementos esenciales para su presentación. Por lo tanto, extrajimos los elementos más originales eligiendo diversos fragmentos y eliminando los pasajes relativos a los conocimientos más clásicos. El lector interesado en este texto encontrará en el informe de referencia desarrollos que no han perdido ni un ápice de interés.

\section{BIBLIOGRAFÍA}

Chesnay, M. (2013). "L'Arbre des causes: Histoire des fondements et usages de la méthode". En C. Teiger y M. Lacomblez (Se) Former pour transformer le travail (pág. 470-478). Laval, Presses de l'Université Laval.

Faverge, J. M. (1967). Psychosociologie des accidents du travail. P.U.F. París.

Faverge, J. M. (1972). Les accidents du travail. En M. Reuchlin (Ed.). Traité de Psychologie Appliquée (pp...223-262). t. 3. P.U.F.: París.

Lahy, J.-M. y Pacaud Korngold S. (1936). "Recherches expérimentales sur les causes psychologiques des accidents du travail”. Le Travail Humain, Serie B, 1, 1-73. 
Leplat, J. (1985). Erreur humaine, fiabilité humaine dans le travail. A. Colin: París.

Leplat, J. (2013). “Contribution de S. Pacaud à l'étude des accidents”. En R. Ouvrier-Bonnaz y A. Weill-Fassina (2013). Suzanne Pacaud (1902-1988). De la psychotechnique à l'ergonomie (coord.) (pp. 252-293). Toulouse: Octares/Lettropolis. (edición electrónica).

Metz, B. ; Lederman, S. (1960). “Les accidents du travail et l'alcool”. Population, 2 (sin paginación). Turbiaux, M. (1982-3). J.M. Lahy (1872-1943) Essai de bio-bibliographie. Bulletin de psychologie, 362, 969-985.

\section{NOTAS}

1. Esta numeración y las que siguen remiten al documento de referencia del que se ha extraído el presente artículo.

\section{AUTOR}

\section{JACQUES LEPLAT}

Groupe de Recherche et d'Etude sur l'Histoire du Travail et de L'Orientation (GRESHTO), Centre de Recherche sur le Travail et le Développement (CRTD), Conservatoire National des Arts et Métiers (CNAM) , 41, Rue Gay Lussac 75005 Paris, France jacques.leplat@wanadoo.fr 